Carnets de géographes

GÉOGRAPHES

$3 \mid 2011$

Les géographies des enfants et des jeunes

\title{
Migrations et division sociales de l'espace en Toscane : structures et dynamiques
}

David Frantz

\section{(2) OpenEdition}

\section{Journals}

Édition électronique

URL : http://journals.openedition.org/cdg/2499

DOI : $10.4000 /$ cdg. 2499

ISSN : 2107-7266

Éditeur

UMR 245 - CESSMA

\section{Référence électronique}

David Frantz, «Migrations et division sociales de l'espace en Toscane : structures et dynamiques », Carnets de géographes [En ligne], $3 \mid$ 2011, mis en ligne le 01 décembre 2011, consulté le 25 septembre 2020. URL : http://journals.openedition.org/cdg/2499; DOI : https://doi.org/10.4000/cdg.2499

Ce document a été généré automatiquement le 25 septembre 2020

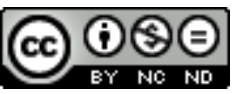

La revue Carnets de géographes est mise à disposition selon les termes de la Licence Creative Commons Attribution - Pas d'Utilisation Commerciale - Pas de Modification 4.0 International. 


\title{
Migrations et division sociales de l'espace en Toscane : structures et dynamiques
}

\author{
David Frantz
}

1 L'objectif de cette thèse est d'étudier les rapports entre d'une part la division sociale de l'espace - mesurée par la distribution spatiale des différentes professions et catégories sociales (PCS) - d'une formation sociale du Centre capitaliste, et d'autre part la répartition des populations migrantes dans cette dernière. L'hypothèse principale stipule que la division spatiale des populations immigrées tient compte des mutations économiques et de la dynamique de la division sociale de l'espace hôte, conformément au concept $d$ ' "immigration post-fordiste" du sociologue italien E. Pugliese.

2 Le choix d'un plan classique allant de la petite à la grande échelle (au sens géographique) a permis d'adopter une présentation qui va du plus simple et du plus théorique à la complexité de la réalité. De l'exposition synthétique de l'analyse marxiste du capitalisme avec l'importance donnée à la division sociale en classes sociales, aux liens entre espace, migrations internationales et capital (marché du travail, mondialisation, droits), à la division sociale de l'espace urbain définie comme espace de la lutte des classes tant pour les classes sociales que pour l'insertion urbaine des populations immigrées, la première partie de l'étude s'est attachée à exposer les fondements de la division sociale de l'espace sur la base de la théorie de Marx.

Ensuite, l'exposition des contextes géographique et social de l'Italie et de la Toscane (parties 2 et 3) a permis de décrire le cadre dans lequel se déroule l'immigration des années 1980 aux années 2000. Les déséquilibres territoriaux (le principal étant la division Nord/Mezzogiorno), la crise démographique, la flexibilisation-précarisation du travail ainsi que la suburbanisation structurent les mutations socio-économiques et spatiales de la Péninsule. C'est dans ce contexte national post-fordiste qui s'appuie sur une vulnérabilisation juridique (titres de séjour, emplois formels, accès au logement, etc.) que l'immigration - récente, relativement massive et diversifiée - est reléguée au travail et à l'espace les plus difficiles et précaires. En Toscane, région définie dans les 
années 1980-1990 comme faisant partie de la "Troisième Italie" (districts industriels et flexibilité du travail), la distribution spatiale de l'immigration est conforme aux déséquilibres démographiques et économiques du territoire, Florence étant le pôle principal d'accueil des nouvelles populations. Les modèles territoriaux du travail immigré proposés par le sociologue M. Ambrosini vérifient les liens entre les secteurs d'activité, la forme du travail (déclaré ou informel), les nationalités immigrées et leur sexe : les modèles de l'industrie diffuse (districts) et de l'économie métropolitaine (care féminin) représentent les deux principaux territoires de l'immigration.

4 A une échelle plus restreinte, l'analyse de la division sociale au sein de l'espace intraurbain florentin a permis d'établir les correspondances entre la structuration sociospatiale de la capitale régionale et les divers systèmes migratoires (parties 4 et 5). Dans un premier temps, l'analyse géo-historique de l'espace florentin a confirmé l'héritage de la division de la ville entre un Est bourgeois et un Ouest populaire dont les prémisses ont été mis en place par la planification urbaine de la seconde moitié du XIX ${ }^{e}$ siècle. Depuis cette période, la ville a constitué un enjeu social, économique et spatial pour la bourgeoisie florentine, qui s'est vérifié à la fin du $\mathrm{XX}^{\mathrm{e}}$ siècle au travers de l'expansion urbaine et de la suburbanisation, de la désindustrialisation et de la tertiarisation, les grandes lignes de la division socio-spatiale étant confirmées par l'affirmation et la concentration des nouvelles classes moyennes- supérieures (cadres, professions libérales). La distribution des populations étrangères repose principalement sur cette division Est/Ouest, le centre historique de la ville étant un espace plus mixte quoiqu'en voie d'embourgeoisement. Cette division a d'abord été vérifiée en distinguant les étrangers suivant leur provenance entre pays du Centre (ou pays "riches") et pays de la Périphérie (ou pays "pauvres"), et ensuite suivant la spécialisation fonctionnelle des populations de ces derniers (les "immigrés" proprement dit). Ainsi, leur espace résidentiel correspond aux quartiers populaires de l'ouest de la ville, plus sous une forme diffuse que de concentration (sauf pour les Chinois), tandis que l'espace des communautés employées dans le care et la domesticité (Philippines, Sri Lankais) est conforme à celui de leurs familles-employeurs des classes moyennes-aisées chez qui elles habitent. En outre, une enquête par questionnaires et entretiens sur le marché touristique de San Lorenzo a permis d'étudier les dynamiques de rapports de travail entre populations migrantes et autochtones dans un espace de travail spécifique du centre de Florence.

5 Enfin, la complexité de la réalité migratoire comme interface entre migration et contexte d' "accueil" a été illustrée par la situation des Roms à Florence (partie 6). Cette population présente en effet des caractéristiques socio-économiques, juridiques et migratoires qui, dans le contexte xénophobe du passage au XXIè siècle en Italie, interrogent de manière accrue les rapports entre formations sociales. Entretiens et terrain ont été nécessaires afin de comprendre les alternatives mises en place par les autorités locales dans le but de dépasser l'habitat de relégation des Roms (les campi nomadi), et par conséquent les difficultés de l'action publique locale à apporter des réponses à l'insertion des populations migrantes.

Fiche informative

Lien électronique vers la thèse

http://tel.archives-ouvertes.fr/tel-00559858/fr/ 


\section{Discipline \\ Géographie}

\section{Directeur}

Petros Petsimeris

\section{Université}

Université de Caen Basse-Normandie

Membres du jury de thèse, soutenue le 11 décembre 2010

- Jean-René Bertrand, Professeur émérite de géographie, Université du Maine, rapporteur

- Sergio Conti, Professore ordinario, Università degli Studi di Torino, rapporteur

- Marco Costa, Professore ordinario, Università di Trento

- Jean-Marc Fournier, Professeur de géographie, Université de Caen-Basse Normandie

- Robert Hérin, Professeur émérite de géographie, Université de Caen-Basse Normandie, président du jury

- Petros Petsimeris, Professeur de géographie, Université de Paris I PanthéonSorbonne, directeur de Thèse

\section{Contact de l'auteur}

david.frantz[at]unicaen.fr

\section{BIBLIOGRAPHIE}

AMBROSINI M. (2008) Sociologia delle migrazioni, Bologna, Il Mulino, 294 p.

BAGNASCO A., TRIGILIA C. (1993) La construction sociale du marché. Le défi de la troisième Italie, Paris, ed. de l'ENS-Cachan, $284 \mathrm{p}$.

BIHR A. (2001) La reproduction du capital : prolégomènes à une théorie générale du capitalisme, Lausanne, Page Deux, 2 vol. , 347 et 359 p.

BORTOLOTTI F. (a cura di)(1994) Il mosaico e il progetto. Lavoro, imprese, regolazione nei distretti industriali della Toscana, Milano, Ires Toscana / Franco Angeli, 400 p.

BRUN J., RHEIN C. (dir)(1994) La ségrégation dans la ville : concepts et mesures, Paris, L'Harmattan, $258 \mathrm{p}$.

DUMÉNIL G., LEVY D. (2003) Economie marxiste du capitalisme, coll. "Repères" n 349, Paris, La Découverte, $121 \mathrm{p}$.

Fondazione Michelucci (a cura di)(2008) Rom e Sinti in Toscana 2007. Tra superamento dei campie nuove baraccopoli, Firenze, Regione Toscana - Fondazione Michelucci, 33 p. 
FRANTZ D. (2011) «L'action publique locale au défi de l'habitat des Roms à Florence (Italie) : en finir avec les campi nomadi. Variété et fragilité des expériences (années1990-2000) », Géocarrefour, vol. 86, pp. 265-275

GAUDEMAR J.P. de (1976) Mobilité du travail et accumulation du capital, Paris, éd. François Maspero, $272 \mathrm{p}$.

PUGLIESE E. (2002) L'Italia tra migrazioni internazionali e migrazioni interne, Bologna, Il Mulino, $145 \mathrm{p}$.

REYNERI E. (1998) «The role of the underground economy in irregular migration to Italy : cause or effect? », Journal of ethnic and migration studies, a. 24, $n^{\circ} 2$, pp. 313-331

INDEX

Thèmes : Carnets de soutenances 